\title{
Féeries
}

Études sur le conte merveilleux, XVII $-\mathrm{XIX}{ }^{\mathrm{e}}$ siècle

$16 \mid 2020$

Le conte, les mythes antiques, la Bible

\section{Confondre les sages : échos évangéliques dans un conte facétieux de Straparola}

But God chose what is foolish in the world to shame the wise: About a comic tale from Straparola's Pleasant Nights (IX, 5) and its evangelical resonance.

\section{Patricia Eichel-Lojkine}

\section{OpenEdition}

Journals

Édition électronique

URL : https://journals.openedition.org/feeries/2586

DOI : $10.4000 /$ feeries. 2586

ISSN : $1957-7753$

Éditeur

UGA Éditions/Université Grenoble Alpes

Édition imprimée

ISBN : 978-2-37747-194-2

ISSN : 1766-2842

Référence électronique

Patricia Eichel-Lojkine, « Confondre les sages : échos évangéliques dans un conte facétieux de Straparola », Féeries [En ligne], 16 | 2020, mis en ligne le 07 janvier 2021, consulté le 10 décembre 2021. URL : http://journals.openedition.org/feeries/2586; DOI : https://doi.org/10.4000/feeries.2586

Ce document a été généré automatiquement le 10 décembre 2021.

(c) Féeries 


\title{
Confondre les sages : échos évangéliques dans un conte facétieux de Straparola
}

But God chose what is foolish in the world to shame the wise: About a comic tale from Straparola's Pleasant Nights (IX, 5) and its evangelical resonance.

\author{
Patricia Eichel-Lojkine
}

1 Les appels à l'humilité sont nombreux dans la Bible, à commencer par les invocations pressant le Tout-Puissant de rabaisser ces orgueilleux qui se permettent d'oppresser son peuple: «Lève-toi, juge de la terre, retourne aux orgueilleux leur salaire [...], ils déblatèrent, ils ont le verbe haut, ils se rengorgent, tous les malfaisants [...], Yahvé sait les pensées de l'homme et qu'elles sont du vent ${ }^{1}$.» Fiers de leur savoir et de leur pouvoir, ces hommes ont oublié leur totale vacuité. Encore plus centrales dans la tradition chrétienne sont les paroles de saint Paul (Paul de Tarse). L'Évangile doit être annoncé indifféremment "aux savants comme aux ignorants» (Rm 1,14). Une distinction claire doit être faite entre scientia et sapientia, entre ceux qui manient « la sagesse du langage » (la rhétorique) et ceux qui parlent « le langage de la croix » (1 Cor 17-31). Dans cette épître aux Corinthiens, des formules des livres prophétiques de la Bible hébraïque sont citées : ainsi « la sagesse des sages se perdra, et l'intelligence des intelligents s'envolera ${ }^{2}$ » de sorte que le raisonneur, l'instruit (litteratus), le docteur de petits riens (doctor parvulorum) seront bientôt réduits à néant ${ }^{3}$. D'ailleurs, ce qui a été caché aux sages et aux intelligents n'a-t-il pas été révélé aux enfants (Matt 11,25) ? À l'authentique sagesse humaine, qui passe nécessairement par l'humilité, s'oppose la suffisance du docteur plein de son vain savoir, facilement pris à sa propre astuce.

Il s'agit de confondre les sages et les forts et, pour cela, Dieu a choisi ce qu'il y a de fou et ce qu'il y a de faible dans le monde ${ }^{4}$ - comme le rappelle Érasme à la fin de la célèbre déclamation de Dame Folie. Alors que le paulinisme revient sur le devant de la scène humaniste et évangélique, on observe que des auteurs d'histoires facétieuses en vernaculaire, qui destinent leurs publications à un public plus mêlé qu'Érasme, ne s'interdisent pas non plus l'usage de références qui font partie de la culture biblique 
commune: quel est alors leur but, quel est l'effet produit? Comment un thème évangélique peut-il se combiner avec des motifs folkloriques traditionnels? N'y auraitil pas davantage de perméabilité entre le répertoire des anecdotes de source biblique et celui des contes à rire que ce qu'on imaginerait a priori ? Comment éviter que l'histoire piquante ne soit contaminée par la lourdeur didactique des exempla? Pourquoi encadrer par une référence paulinienne un récit, si réconfortant pour les auditeurs, où les faibles l'emportent sur les forts?

Ces questions émergent à la lecture d'un conte de la collection des Piacevoli Notti (Les Facétieuses Nuits) de Straparola moins étudié que d'autres, la $5^{\mathrm{e}}$ fable de la IX $\mathrm{X}^{\mathrm{e}}$ iit: «Les Florentins et les Bergamasques assemblent leurs docteurs pour disputer les uns contre les autres $[. . .]^{5}$.» L'histoire articule au thème de la confusion des sages un dispositif renvoyant à une pratique de l'université médiévale: la disputatio ou joute oratoire, qui commence alors à inspirer les satiristes.

\section{Masques et bergamasques}

4 Inutile de rappeler que le recueil vénitien de Giovan Francesco Straparola fut un bestseller italien absolu dans la seconde moitié $\mathrm{du} \mathrm{xvI}^{\mathrm{e}}$ siècle et qu'il fut rapidement traduit en français par Jean Louveau (1560) et Pierre de Larivey (1572/1585) ${ }^{6}$. La lecture des Facétieuses Nuits dans les salons parisiens à la fin du xvII ${ }^{\mathrm{e}}$ siècle inspira plus d'un conte à $\mathrm{M}^{\mathrm{me}}$ de Murat, $\mathrm{M}^{\mathrm{me}} \mathrm{d}$ 'Aulnoy et Perrault.

5 Il arrive que "des savants" soient «vaincus par les hommes qui n'avaient point de lettres car ceci se voit clairement en l'écriture sainte, où les apôtres, simples et abjects, confondirent la sapience de ceux qui étaient réputés sages et prudents 7 .» D'entrée de jeu, le conteur du récit-cadre, le seigneur Ferrier (il signor Feriero), convoque l'opposition paulinienne entre scientia et sapientia. La réhabilitation évangélique des simples va permettre de subvertir la hiérarchisation entre les doctes dédiés aux choses de l'esprit et les hommes grossiers conditionnés par leurs appétits et la lourdeur de leur corps. Cette référence à l'épître aux Corinthiens, qui peut aussi faire venir à l'esprit l'épisode de Jésus enfant frappant d'étonnement les docteurs ${ }^{8}$, rejoint le thème religieux de la vanité (dans les deux sens de nullité et d'orgueil) de l'homme par rapport à la grandeur de Dieu, un thème littéraire fréquent $a u \mathrm{Xv}^{\mathrm{e}}$ et au début du $\mathrm{XVI}^{\mathrm{e}}$ siècle ${ }^{9}$.

6 Le thème moral et évangélique, particulièrement bien adapté au regard de la réputation des Florentins si fiers de leur situation au centre de la culture, est ici couplé à un motif narratif typique, la dispute entre deux cités rivales ${ }^{10}$. Ce motif va prendre tout son sel d'une conclusion paradoxale : dans la rivalité opposant des « rustiques » de Bergame à des citadins de Florence, les premiers vont l'emporter et cela, sans même disputer. Depuis plus d'un siècle, les Bergamasques correspondent au type du travailleur fruste, sans prétention, à la psychologie simple, s'exprimant en dialecte ${ }^{11}$. Ils ont donc le physique de l'emploi pour jouer le rôle de "ceux qui sont matériaux et grossiers ", écrasés par la supériorité sociale des «hommes savants et lettrés » ${ }^{12}$. On aura repéré également la composante personnelle sous-jacente: Straparola de Caravaggio, probablement d'extraction modeste, est originaire d'un village de Lombardie proche de Bergame. 
7 Dans cette nouvelle, l'originalité de l'invention littéraire tient à la scénarisation de l'affrontement qui procède par étapes, jusqu'à la résolution de la compétition :

8 1. Une altercation a lieu : un commerçant florentin déverse son mépris hautain sur les Bergamasques, traités d'ignares. Sans se laisser démonter, un marchand bergamasque répond, d'abord, en opposant le vain art rhétorique des Florentins à l'esprit pratique des siens, puis déclare, bravache, que dans sa cité, les lettres (latines, s'entend) sont un bien partagé par tous : "Si venons à bien tout considérer, il n'y a aucun de notre nation, quel qu'il soit, grand ou petit, qui n'ait quelques lettres $[. . .]^{13}$.» L'échange agressif débouche sur un défi en forme. Pour que les docteurs des deux villes puissent faire la preuve de leur excellence et se mesurer, un autre Bergamasque présent fait la proposition d'organiser une dispute solennelle, aux calendes de mai, en sa bonne ville (le hasard décidant du lieu).

9 2. L'honneur de Bergame est en jeu: c'est pourquoi les marchands se tournent vers leurs docteurs en théologie. Connaissant la réputation de savoir des Florentins, ils inventent un stratagème pour les intimider, dans l'espoir qu'ils arrivent démoralisés à la confrontation publique. L'astuce consiste à dépêcher à leur rencontre des doctes déguisés en paysans ${ }^{14}$, mêlés aux gens des champs. Le récit avance au gré de la chevauchée des Florentins sur la route. Leurs salutations et leurs questions concrètes, formulées en italien («Combien y a-t-il encore d'ici à Bergame?»), leur attirent à chaque fois des réponses... en latin ${ }^{15}$. On pense au dernier mouvement de cet autre conte de Straparola - «Costantino Fortunato » $(\mathrm{XI}, 1)$ - qui a inspiré à Perrault son Maître Chat ou Chat botté. Alors que Costantino conduit son épouse Elisetta, la fille du roi, vers son château supposé, la suite de la princesse s'arrête pour demander à un groupe de cavaliers, puis aux autres personnes croisées en chemin, qui est leur seigneur et ces derniers leur font à chaque fois la réponse exigée par la chatte ${ }^{16}$. Dans le texte de Perrault, le carrosse du roi fait route vers le château de l'ogre et s'arrête successivement auprès de faucheurs, puis de moissonneurs: on leur demande à qui appartiennent ces terres alentour et on reçoit à chaque fois la même réponse préparée par le chat (" au marquis de Carabas »). Au terme d'un trajet ponctué par des échanges faussement spontanés, des voyageurs pourtant regardants se trouvent dupés.

10 Mais à Bergame, aux questions prosaïques sur la longueur du chemin restant à parcourir jusqu'à la ville, succèdent bientôt de véritables débats philosophiques : «[...] et argumentaient les Bergamasques si doctement que les Florentins ne savaient quasi que répondre ${ }^{17}$.» Quand ils arrivent à l'hôtellerie, un garçon d'écurie, puis le propriétaire les accueillent : ces derniers semblent aussi trouver tout naturel de leur parler latin. Et voilà que les docteurs assistent à une scène encore plus étrange : une servante (une jeune religieuse déguisée) se met à deviser théologie devant eux, avec des arguments appuyés par un pseudo-boulanger qui arrive incidemment sur les lieux.

11 3. La ruse fonctionne mieux que prévu: les Florentins renoncent tout de bon à la disputatio, en se fondant sur un raisonnement $a$ fortiori :

[...] déterminèrent qu'il serait meilleur s'en retourner, pour ce que si les bonnes gens des champs, les hôteliers, les garçons d'étable et les femmes sont de si profonde doctrine, que devait-ce être de ceux de la ville, où sont les hommes consommés de science, et qui ne pensent à autre chose qu'à leur continuelle étude $^{18}$ ? 
Straparola, s'inspirant lointainement de Morlini ${ }^{19}$, construit son récit autour d'un bon tour qui tourne à la confusion des arrogants, c'est-à-dire des professeurs (« in vituperio de' dottori firentini $\left.i^{20} »\right)$ et nous fait attendre une dispute solennelle qui ne viendra jamais.

\section{Une histoire en trompe-l'œil ?}

13 Les doctes latinistes sont ridiculisés, humiliés par des hommes de peu, plus rusés qu'eux : le récit de "l'astuce des Bergamasques » suscite pour finir la " grande risée » des devisants-auditeurs du récit-cadre. Ne nous laissons pas prendre aux apparences pour autant : les « rustiques ", s'ils sont présents sur scène, le sont à titre de figurants, font tapisserie en quelque sorte. Les échanges se font bien entre des doctes des deux villes, mais pas dans le cadre attendu d'une disputatio, plutôt sur le mode d'une guerre psychologique où ceux qui avancent masqués, sous couvert d'une identité usurpée, ont un avantage psychologique certain sur ceux qui se présentent sous leur vraie identité. Un paysan pauvre, une identité usurpée, des habits empruntés : c'est encore une fois «Costantino Fortunato » et le « Maître Chat » qui viennent à l'esprit.

14 L'anecdote ne ridiculise donc que superficiellement les doctes (mais vraiment les arrogants). Ce sont les plus savants des Bergamasques qui ont été appelés au secours par des marchands de retour chez eux après un défi lancé un peu imprudemment ${ }^{21}$. Merveilleuse cité où les marchands et les savants se donnent la main et où tous se réjouissent de la compétition à venir! Les doctes ont l'intelligence de savoir qu'ils seraient probablement vaincus dans une disputatio à la loyale. Ils tirent donc les ficelles :

- d'abord en attribuant des identités fictives aux clercs, grammairiens, maîtres ès arts, religieuses qu'ils décident d'envoyer en mission (en créant donc en quelque sorte un «bureau des légendes »);

- ensuite, en s'avisant de déplacer l'accent, pour ainsi dire, de l'affrontement solennel final vers les étapes intermédiaires, dans l'espoir de miner le moral de l'adversaire : justement parce que ces échanges banals ne prennent pas l'allure de confrontations entre deux camps, rien n'éveille la méfiance des Florentins qui se projettent dans l'épreuve à venir, et ne voient pas que le match a déjà commencé dès le premier face-à-face. Il s'agit en apparence de simples rencontres anodines avec des gens de peu qu'on croise par hasard sur sa route, à qui on demande un renseignement. Et voici que ces rustres s'expriment dans un latin sans faute. Concentrés sur le grand débat à venir, ils n'imaginent pas que tout se joue en amont, dans ces moments informels, qui sont la clé du retournement de situation.

En fait, les Bergamasques ont pensé à répartir sagement leurs forces entre cette première étape et la grande bataille à venir, dans le cas, probable, où celle-ci aurait effectivement lieu :

À raison de quoi, ayant rassemblé les plus doctes de la cité, grammairiens, rhétoriciens, légistes, canonistes, philosophes, théologiens et tout autre genre de docteurs, en choisirent les meilleurs pour demeurer en la cité, comme leur rempart et forteresse contre les Florentins. Puis firent vêtir les autres à la villageoise et les envoyèrent hors la ville, sur le grand chemin par lequel devaient passer les Florentins, leur enchargeant que toujours ils parlassent à eux latin ${ }^{22}$.

Les docteurs bergamasques inventent la guerre d'intimidation pour impressionner l'ennemi avant l'engagement ${ }^{23}$. L'arme fatale, ce sont des paroles en latin - une langue que les clercs, les magistri et les docteurs des deux côtés maîtrisent sans doute (même si 
c'est avec moins de compétence à Bergame qu'à Florence), mais qu'on ne s'attend pas à entendre dans la bouche de tout un chacun, en dehors des usages liturgique, théologique et scolaire. Les Florentins croient à un phénomène surnaturel. Ils sont «bien étonnés ${ }^{24}$ » et demeurent bientôt « tous $\operatorname{confus}^{25}$ ", comme les docteurs devant le jeune Jésus, et ne sauraient expliquer ces miracles : «Comment est-il possible que ces hommes grossiers et dédiés à l'agriculture et œuvres rustiques soient tant bien instruits ès sciences humaines ${ }^{26}$ ?» Ils sont abusés parce qu'ils n'ont pas accès au dessous des cartes: ils prennent pour argent comptant le spectacle disposé à leur intention, comme s'il s'agissait d'une scène prise sur vif, sans comprendre que derrière ce premier plan, il y a un second plan caché à leur regard ; que derrière la scène, tout se trame dans les coulisses.

L'astuce a changé de camp ${ }^{27}$. Les Bergamasques manipulateurs sont certes du côté de la ruse, mais sans que cela soit incompatible avec le savoir. La nouvelle témoigne d'une sympathie certaine à l'égard de gens du peuple éloignés du centre culturel et artistique qu'est Florence, mais sans "populisme»: Straparola ne figure nullement un vilain faisant mordre la poussière à un docte, mais, pour commencer, deux commerçants qui s'affrontent, puis un groupe de docteurs (florentins) faisant face, sans le savoir, à d'autres lauréats d'un grade universitaire (bergamasques). Nul conflit interne n'est figuré non plus au sein de la cité de Bergame: au contraire, les latinistes (maîtres gradués, docteurs dans les deux droits ou en théologie, clercs, religieuses) travaillent en pleine connivence et concertation avec les ignorants que sont les paysans courbés sur leurs champs et les commerçants voyageant pour affaires. Entre le monde savant, le monde citadin des artisans et le monde paysan, le dialogue est fluide, peut-être parce que tous sont censés avoir une teinture de savoir. Reprenant le motif narratif de la rivalité entre cités, Straparola en profite pour mettre en valeur un contre-modèle par rapport à l'organisation de Florence, avec une communauté bergamasque où personne n'est complètement éloigné des lettres et où les tensions internes entre groupes sociaux sont lissées. Voilà un positionnement original, qu'on ne retrouvera pas exactement chez Des Périers ${ }^{28}$.

\section{Des « pédants joués »?}

18 Ce conte de Straparola s'ouvre sur une déclaration valorisant les simples qui n'ont pas de lettres et se conclut par une représentation ridicule des docteurs florentins qui rentrent au pays la queue basse. La leçon paulinienne rejoint-elle le thème satirique du "pédant joué ", récemment apparu sur la scène théâtrale italienne, comme dans la novellistica? Une comparaison avec des productions contemporaines s'impose pour savoir si la référence paulinienne exhibée est une vraie piste de lecture ou un fauxsemblant.

19 Chez Des Périers, dans l'histoire de la harangère du Petit Pont, une femme du peuple au verbe haut a le dessus sur un "magister crotté » du collège de Montaigu ${ }^{29}$. Mais à la différence d'un Des Périers ou d'un Rabelais, Straparola ne cherche manifestement pas ici à railler la vieille culture scolastique et à jeter le discrédit sur les docteurs ${ }^{30}$. Son usage du bilinguisme latin/vernaculaire est significatif. Il produit des effets comiques dans le texte du fait de l'entrelacement de questions en vernaculaire - faites par les théologiens - et de réponses en latin - faites paradoxalement par des " paysans »: 
[...] mais comme ils voulaient monter dans leur chambre pour se reposer, le maitre du logis se présente à eux, disant: «Excellentissimi domini, placetne vobis ut præparetur cœna? hic enim sunt bona vina, ova recentia, carnes, volatilia et alia hujusmodi. » Les Florentins demeuraient tous étonnés [...] $]^{31}$.

Ici, nul langage hybride ni latin forgé, contrairement à ce qui se passe avec le "pedante $e^{32}$ », un personnage théâtral qui fait son apparition dans les "comédies érudites » italiennes exactement au temps de Straparola ${ }^{33}$. L'emploi du latin commence alors à perdre de son prestige par son association au monde de l'éducation ${ }^{34}$. Le personnage du " pédant ", sans équivalent dans la tradition antique, se définit par un langage et des comportements tendant vers l'affectation et l'artifice : c'est tantôt un clerc (maître d'école) ou un régent de collège au savoir limité, tantôt un docteur universitaire infatué de son érudition livresque. Dans les deux cas, il entretient, par défaut ou par excès, un rapport faussé, distordu au savoir ${ }^{35}$, qui se traduit par le fait qu'il amène sur scène, dès qu'il ouvre la bouche, une mixture franco-latine à effet comique. Lorsque le docteur jargonnant le latin est en fait un jeune galant florentin qui se fait passer pour un éminent médecin, comme dans La Mandragore (1526) de Machiavel (probablement connue de Straparola), l'effet comique est démultiplié. À la fin du siècle, Pierre Charron écrira :

En toute langue et nation, pédant, clerc, magister sont mots de reproche, faire sottement quelque chose, c'est le faire en clerc: ce sont gens qui ont la mémoire pleine du savoir d'autrui, et n'ont rien de propre ${ }^{36}$.

Or Straparola n'utilise pas ici la ressource comique de prendre un "pedante pour badin » (selon le mot fameux de Montaigne ${ }^{37}$ ). Si, dans la comédie, « le pédant [est la] caricature contemporaine des aberrations auxquelles avait abouti un certain type de culture humaniste ${ }^{38} "$, ce n'est pas ici le projet du conteur que d'exhiber ces aberrations.

Ce conte s'inscrirait-il davantage dans le sillage des représentations satiriques du sacerdos indoctus, ce prêtre demi-lettré content de sa personne, qui cherche à briller au milieu des ignorants? L'invention de ce type est alors toute récente. Elle dérive d'une critique d'obédience humaniste et réformée prenant pour cible des hommes d'Église sans culture ${ }^{39}$. Toutefois, la "fable» de Straparola ne montre aucun étalage d'une fausse science : les discours théologiques relèvent certes de l'« intox » du point de vue de la communication, mais rien ne dit qu'ils sont de la « poudre aux yeux » du point de vue du contenu. Dans la fable précédente des Facétieuses Nuits ${ }^{40}$, on trouvait un faux savant de cet ordre avec "Papiro Schizza pedante»-une histoire reprise par Des Périers ${ }^{41}$. Un prêtre aussi suffisant et méprisant qu'il est mauvais latiniste enseigne un vocabulaire latin extravagant à un jeune étudiant de Padoue et prétend même le corriger devant son père quand il avance des objections. Or le jeune homme en sait plus que cet examinateur farcesque. Tout en feignant la naïveté, il imagine, pour finir, de jouer sur les mots de faux latin inculqués par le prêtre pour fomenter une vengeance. Il attache une torche enflammée à la queue d'un chat pour mettre le feu à sa maison, à la manière de Samson se vengeant des Philistins ${ }^{42}$. Parce que le prêtre inculte était capable de sacrifier l'avenir d'un étudiant pour sa gloriole personnelle, celui-ci semble en droit de mettre un terme à sa capacité de nuisance.

Mais contrairement à ce prêtre imposteur puni, les docteurs florentins sont véritablement instruits : ils sont finalement dupés par d'autres doctes (déguisés), non par des ignorants ou par des demi-savants. S'ils ressemblent aux «pédants» de 
théâtre ${ }^{43}$, s'ils sont partiellement conformes au tableau sévère des professeurs brossé ultérieurement par Pierre Charron ${ }^{44}$, c'est par un autre trait : leur vanité.

Face à eux, les docteurs bergamasques semblent jouer parfaitement leur rôle d'élite sociale et intellectuelle, tout en restant à l'arrière-plan du récit. Ils évitent une disputatio qui aurait sans doute tourné à leur désavantage et apparaissent donc comme des personnages entièrement positifs : ils sont à l'origine d'un bon tour qui met à mal le stéréotype de l'infériorité culturelle des "bouseux ». En prêtant une main secourable aux faibles, ils rendent possible ce retournement de comédie où le puissant est défait par le déshérité et où l'effet de comique verbal repose sur un mélange de vernaculaire et de latin. Ce dénouement s'inscrit dans une tradition narrative qui remonte aux fabliaux ${ }^{45}$, tout en trouvant maintenant un relais dans le courant savant évangélique : «[...] ce qu'il y a de faible dans le monde, voilà ce que Dieu a choisi, pour couvrir de confusion ce qui est fort $[. . .]^{46}$.»

En fait, la nouvelle n'oppose pas, ou pas seulement, les Florentins doctes et fort contents d'eux-mêmes aux Bergamasques sages (c'est-à-dire astucieux) malgré leur ignorance. En donnant le beau rôle aux docteurs bergamasques, elle ne laisse percevoir aucune raillerie aigre sur les savants ${ }^{47}$, aucune défiance de la formation scolastique et des arts libéraux non plus. La cité de Bergame montre une société élitiste équilibrée, où la différence d'éducation - c'est-à-dire les degrés dans la maîtrise de la langue liturgique et savante et des matières théologiques - produit une hiérarchie acceptée par tous et profitable à tous. L'image de l'université reste intacte ${ }^{48}$. Pour finir, les docteurs en question ne font pas figure de savants éloignés des réalités pratiques ou coupés du reste des groupes sociaux - un autre reproche fréquent en direction des gens instruits. On les voit imaginer des techniques d'intimidation, presque des pièges de guerre, pour faire fuir l'adversaire et préserver ainsi l'honneur de la cité tout entière. L'épisode qui fait tout basculer demande à être regardé avec soin :

Ils ne furent guère qu'une jeune fille arriva, laquelle véritablement était religieuse, de grand savoir et bonne doctrine [...]. Enfin se mirent à deviser avec elle, qui, tombant sur la théologie en parla tant doctement qu'il n'y eut aucun qui ne l'êt en grande réputation. Tant qu'elle disputait, arriva un vêtu en boulanger, tout blanc de farine, lequel les entendant disputer, se mit du côté de la fille, interprétant les écritures saintes avec tant de doctrine que tous les docteurs florentins affirmaient n'avoir jamais ouï mieux parler ${ }^{49}$.

La figure de la femme lettrée donne le coup de grâce aux Florentins : dans le monde à l'envers qu'est cette cité où les laboureurs, les valets et les boulangers parlent latin, elle représente le renversement de l'ordre social poussé jusqu'à son dernier terme. Les discussions auxquelles elle prend part n'ont rien de ridicule, ni sur le fond, ni dans la forme, mais attestent d'une science sûre, d'une formation achevée. Avec ce personnage de jeune intellectuelle maitrisant l'art rhétorique et les points de doctrine et s'exprimant parfaitement dans la langue savante, Straparola prend le contre-pied du stéréotype du "pédant» jargonnant. La joute théologique en règle a bien lieu finalement, mais entre des docteurs en théologie florentins et une religieuse bergamasque déguisée en servante. Dans cette disputatio, les participants jouent certes un rôle, mais comme on s'investirait dans un "serious game ». C'est précisément grâce à ce léger détachement, à cette conscience que la « dispute » relève d'un jeu de rôle codé et d'un théâtre qu'ils l'emportent ${ }^{50}$. Les Bergamasques inventent le paradoxe sur le comédien avant la lettre. 
27 En conclusion, la lecture de Straparola et de Des Périers nous confirme la présence du thème chrétien de l'orgueil et de la vanité dans les contes à rire du $\mathrm{XVI}^{\mathrm{e}}$ siècle. Cette topique a été récemment ravivée par l'approche érasmienne (la satire de la "philautie», de l'amour de soi qui aveugle) et par le courant évangélique (le rabaissement du «cuider", de la présomption, dans toute l'œuvre de Marguerite de Navarre). De manière significative, l'histoire de Papiro Schizza (chère à Des Périers) s'ouvre sur une critique de la "superbia ${ }^{51}$ ». Parallèlement, la satire des "pédants » commence alors à se faire une place dans le théâtre et les écrits satiriques avec la représentation de magistri risibles. Cependant, l'histoire des Bergamasques de Straparola ne provoque pas tout à fait le même rire de rejet que l'évocation des premiers maîtres de Gargantua, ou encore l'arrivée sur scène du "pedante » au latin bouffi dans les comédies italiennes. La raison en est double: les docteurs florentins, purs produits de l'université médiévale, sont impeccables sur le plan du savoir; il ne sont pas non plus vaincus par "des hommes qui n'avaient point de lettres », comme annoncé au lecteur, mais par d'autres docteurs honorables, de bon calibre. Sous cet aspect, la citation biblique initiale est à la fois une vraie et une fausse piste : ceux qui attendent une critique évangélique des savants en faveur des simples en seront pour leurs frais, mais ils applaudiront néanmoins au spectacle d'hommes sûrs de leur supériorité, trahis par leur propre suffisance et arrogance ${ }^{52}$.

Sur un plan plus général, la réussite de la nouvelle IX, 5 tient à la conjonction de trois ingrédients, dont deux ont des résonances bibliques : la victoire du faible sur le fort (David contre Goliath, Gédéon contre Madian) ; la supériorité paradoxale du simple sur le savant (ut confundat sapientes) ; la rivalité entre cités figurée par une joute oratoire. Straparola retravaille de manière originale chacun de ces trois thèmes. Il redonne des couleurs au premier en figurant une collectivité soudée (les Bergamasques) au-delà des différences sociales. Il renouvelle le deuxième en mettant en scène de faux simples. Il parodie le troisième en éludant le motif de la "dispute " (la grande disputatio entre Florentins et Bergamasques n'aura pas lieu) pour le réintégrer à un autre niveau, avec l'épisode de la nonne théologienne: car ce qui surprend le plus les Florentins, c'est finalement la vision surnaturelle, dans une auberge de Bergame, d'une "femme de profonde doctrine ", d'une nouvelle Catherine de Sienne qui n'a rien d'une femme savante ridicule ${ }^{53}$.

\section{NOTES}

1. Mes remerciements vont à Pierre-Emmanuel Moog pour sa relecture minutieuse et ses judicieux conseils.

Ps 94 (93),2.4.11 (La Bible de Jérusalem, Paris, Cerf, 1998). Vulgate : «Elevare qui judicas terram redde vicissitudinem superbis [...], fluent loquentes antiquum/garrient omnes qui operantur iniquitatem [...], Dominus novit cogitationes hominum qui vance sunt.» Les cent cinquante psaumes de la Bible hébraïque ont été intégrés dans la liturgie et la culture chrétienne dès le IV siècle.

2. Is 29,14 . Vulgate : "[...] peribit enim sapientia a sapientibus ejus et intellectus prudentium ejus abscondetur. » Paraphrasé dans 1 Cor 1,19. 
3. Is $33,18:$ : [...] "Où est celui qui comptait ? où est celui qui pesait?/ où est celui qui comptait les tours ?" [...].» Vulgate : «[...] "ubi est literatus ubi legis verba ponderans ubi doctor parvulorum [...]." » Paraphrasé dans 1 Cor 1,19 .

4. 1 Cor 1,27 . Vulgate : «Sed quae sunt stulta sunt mundi elegit Deus/ ut confundat sapientes et infirma mundi elegit Deus ut confundat fortia [...]. »

5. Nous citons d'après Les Facétieuses Nuits de Straparole, trad. fr. P. de Larivey (1585), Paris, P. Jannet, 1857, t. 2 : IX, 5, p. 209-216 (ici, p. 209). Pour un meilleur confort de lecture, nous modernisons l'orthographe et la ponctuation dans les citations en moyen français (à l'exception des titres d'ouvrages). Ital. : "I firentini e $i$ bergamaschi conducono $i$ lor dottori ad una disputa, ed e' bergamaschi con una sua astuzia confondeno i firentini [...]. » Édition italienne moderne de référence : Le Piacevoli Notti, éd. D. Pirovano, Rome, Salerno, 2000, vol. 2 (ici p. 607).

6. Il y eut d'abord une traduction des Notti en deux parties séparées - par Louveau ( $1^{\text {re }}$ partie, Lyon, 1560) complété par Larivey pour la $2^{\mathrm{e}}$ partie - avant la réunion en un volume (Lyon, 1572) et une édition entièrement révisée par Larivey (Paris, Abel l'Angelier, 1585). Voir à ce sujet P. Eichel-Lojkine, Contes en réseaux. L'émergence du conte sur la scène littéraire européenne, Genève, Droz, 2012, p. 117.

7. IX, 5, p. 209, ibid. Ital. : « [...] alle volte s'hanno veduti gli sapienti esser superati da gli uomini illiterati. E questo chiaramente si vede nelle scritture sante, dove gli apostoli semplici e abietti confondevano la sapienza de quelli che erano prudenti e savi. » (p.607)

8. Lc 2,47 .

9. Le projet des Folles entreprises de Pierre Gringore (1475-1539) est, par exemple, d'abaisser des "orgueilleux remplis de ventance/ et pertinax [opiniâtres] en leur fière arrogance,/ présomptueux, pleins de contemption » (Euvres complètes, Paris, P. Jannet, 1858, t. 1, p. 16).

10. Motif folklorique de base : J31.1 Wise men of two rival cities engage a dispute. Voir D. Pirovano, éd. citée.

11. Les éditeurs respectivement italien (D. Pirovano, éd. citée) et anglo-saxon (D. Beecher, University of Toronto Press, 2012) nous renseignent sur les antécédents littéraires de cette nouvelle. Le portrait des Bergamasques provient du chant VII du Baldus de Teofilo Folengo (édition princeps, 1517).

12. IX, 5, p. 209. Ital. : "Quantunque, graziose donne, grandissima sia la disagualianza tra gli uomini saputi e litterati e quelli che sono materiali e grossi [...]. » (p. 607) La caractérisation " uomo materiale e grosso » se trouve dans le Décaméron (III, 8) (note de D. Pirovano).

13. Ital. : «E se ben consideriamo non c'è alcuno tra la gente nostra, ò grande, ò picciolo, che si sia, che non habbia qualche lettera [...]. » (p. 608)

14. Nouveau motif folklorique reconnaissable : K1816.9.1 Wise men disguise as peasants.

15. Ital. : « [...] "Quante miglia ci restano sinon alla città di Bergamo ?" A cui riposero i bergamaschi : "Decem, vel circa." [...]. » (p. 610)

16. XI, 1. Ital. : « Le genti che Elisetta accompagnavano addimandavano: "Di chi siete cavallieri e di chi sono tanti bei armenti ?" E tuti ad una voce rispondevano : "Di messer Costantino." [...]. »

17. IX, 5, p. 212. Ital. : " [...] e sí fortemente argoivano [argomentavano] $i$ contadini bergamaschi, che $i$ dottori firentini non sapevano quasi rispondere. » (p. 610)

18. Ital. : « [...] determinarono il partire esser migliore, percioché se ne gli agricoltori, se ne gli osti, se ne' fanti e nelle femine è tanta profunda dottrina, che saria nella città, dove sono uomini consumatissimi, e che ad altro non attendeno che alli contínovi lor studii ?» (p. 610) Nous soulignons.

19. Straparola a adapté et intégré une partie des nouvelles latines de Morlini précisément dans le second volume de ses Piacevoli Notti (1553). Dans la nouvelle 50 (Novelloe, 1520), le fou Gonella, rencontre, à la porte de Naples, des Napolitaines de basse condition qui n'ont pas leur langue dans leur poche : elles l'impressionnent par leur sens de la répartie et il en déduit, par inférence, que les Napolitains doivent tous être d'une intelligence supérieure («De joculatore: Gonnella qui voluit neapolitanos periclitari». BnF/Gallica: <https://gallica.bnf.fr/ark:/12148/ 
bpt6k1311990.image>). Dans des contes d'Afanassiev, on trouve le même type d'astuce amenant un adversaire puissant (un diablotin) à faire un raisonnement par inférence et à renoncer à une compétition directe où il aurait pourtant certainement eu le dessus (« Sac à malices », «IvankoOurseau »).

20. D. Pirovano, éd. citée, IV, 5, p. 612 : « à la honte des docteurs florentins ».

21. " Ainsi chacun des marchands retourna au lieu de sa demeure, où arrivés, racontèrent le tout à leurs docteurs, lesquels furent fort joyeux de ces nouvelles, proposant faire merveilles au jour assigné. » Ital. : «I mercatanti andarono alle lor città e riferirono il tutto alli lor sapienti, i quai, intesa la cosa, furono molto contenti e apparecchioronsi di far una bella e lunga disputa.»(p.609) Nous soulignons.

22. Ital. : " Onde convocati tutti e’ savi della città, sí grammatichi come oratori sí leggisti come canonisti sí filosofi come teologi e di qualunque dottori, fecero la scelta degli migliori, et quelli ritennettero nella città, accioché fussero la rocca e la fortezza nella disputazione contra firentini.» (p.609) Nous soulignons.

23. Un équivalent militaire serait l'épisode biblique où Gédéon sème la panique dans le camp de Madian avec trois cents hommes munis de trompes et de cruches vides : au son des trompes, des cris et des cruches brisées, les Madianites finissent pas s'entretuer avec leurs épées (Jg 7).

24. Les manifestations d'étonnement sont récurrentes: IX, 5, p. 212 et 213. Ital.: " tutti ammirativi » (p. 610), «tutti sospesi » (p. 611).

25. IX, 5, p. 213. Ital. : « E' bergamaschi come persone sagge e astute s'imaginorono di far sí che firentini restassino confusi e scornati. » (p. 609) Nous soulignons.

26. IX, 5, p. 212. Ital.: "Come è possibile che questi uomini rozzi e dediti all'agricoltura e ad altri rusticani essercizii siano sí ben instrutti [istruiti] delle scienzie umane?» (p. 610)

27. IX, 5, p. 214 : «Ainsi, par leur astuce et finesse, les Bergamasques se firent victorieux sur les Florentins »; « [...] sa courte fable qui fit louer l'astuce des Bergamasques et blâmer la peu caute simplesse des Florentins. » Ital. : «E in tal maniera $i$ bergamaschi con la lor astuzia furono contra firentini vittoriosi»; "[...] e l'astuzia de' bergamaschi fu da tutti lodata e la viltà di firentini biasmata. » (p. 612)

28. Des Périers - qui a lu Straparola et adapte certaines de ses nouvelles - prend soin de distinguer entre les catégories sociales (prélat, bourgeois, gentilhomme) et privilégie les personnages de citadins. Quand ses intrigues mettent en place une confrontation intellectuelle, c'est pour montrer des gens du peuple donnant une leçon à des gens de condition supérieure imbus de leur personne. Voir L. Sozzi, Les Contes de Bonaventure Des Périers. Contribution à l'étude de la nouvelle française à la Renaissance, Genève, Slatkine, 1998, p. 390.

29. Des Périers, Les Récréations et joyeux devis, Lyon, G. Rouillé, 1561 ; éd. moderne : éd. K. Kaprzyk, Paris, STFM, 1980. Ici, Nouvelle LXIII. Voir L. Sozzi, ouvr. cité, p. 391-393.

30. Sur le débat contemporain assez complexe entre scolastique et humanisme, voir E. Rummel, The Humanist-Scholastic Debate in the Renaissance and Reformation, Cambridge, Harvard University Press, 1995.

31. IX, 5, p. 213. Ital. : " [...] e mentre volevano salire su per le scale per riposarsi, il patrone dell'albergo si fece in contro, e disse : "Excellentissimi domini, placetne vobis ut præparetur cœna? hic enim sunt bona vina, ova recentia, carnes, volatilia et alia hujusmodi." Stavano i firentini tutti sospesi né sapevano che dire [...]. » (p. 611)

32. Parmi l'abondante bibliographie sur le sujet, on consultera avec profit J. Royé, « Pédant et bel esprit: la représentation du savant mondain et du mondain savant dans les comédies du $\mathrm{XVII}^{\mathrm{e}}$ siècle ", Littératures classiques, 2005/3, n 58, p. 105-113, et La Figure du pédant de Montaigne à Molière, Genève, Droz, 2008; A. Stäuble, "Parlar per lettera». Il pedante nella commedia del Cinquecento e altri saggi sul teatro rinascimentale, Rome, Bulzoni, 1991 ; F. Gabaude, Les Comédies d'Andreas Gryphius et la notion de grotesque, Berne, Peter Lang, 2004 ; P. De Capitani, Du spectaculaire 
à l'intime. Un siècle de commedia erudita en Italie et en France (début du XVI $I^{e}$, milieu du XVII siècle), Paris, Honoré Champion, 2005.

33. Il Pedante de Francesco Belo (Rome, 1529, rééd. 1538) présente un vieux « pédant » amoureux, au langage pédantesque artificiel mêlant latin et italien. Même type de personnage dans Il Marescalco de l'Arétin (Venise, 1533). Le personnage migre ensuite dans les comédies humanistes françaises dont le premier exemple est La Comedie du sacrifice des professeurs de l'Academie vulgaire senoise... traduicte de langue tuscane (ou Les Abusez) de Charles Estienne (Lyon, 1543), adaptée de Gl'Ingannati (attr. A. Piccolomini).

34. Dans les années 1570, Ronsard parlera du latin comme d'« une langue morte, muette et ensevelie sous le silence de tant d'espaces d'ans, laquelle ne s'apprend plus qu'à l'école par le fouet et la lecture des livres, auxquelles langues mortes il n'est licite de rien innover, disgrâciées du temps, sans appui d'empereurs, ni de rois, de magistrats ni de villes, comme chose morte, laquelle s'est perdue au fil des ans [...].» (Euvres complètes, éd. P. Blanchemain, Paris, P. Jannet, 1858, t. 3, «Préface sur La Franciade touchant le poème héroïque », p. 15-37, ici p. 33). Nous soulignons.

35. Un thème auquel, entre $\mathrm{XVI}^{\mathrm{e}}$ et $\mathrm{XVII}^{\mathrm{e}}$ siècle, donneront encore plus d'ampleur Giordano Bruno (Il Candelaio, 1582), Cyrano de Bergerac (Le Pédant joué, 1654) et Andreas Gryphius (Herr Peter Squentz, 1657 et Horribilicribrifax, 1663).

36. P. Charron, De la Sagesse [1601], liv. I, chap. vi, Paris, Hérissant fils, 1768, p. 60-61 (en manchette : «Pedans»).

37. Essais, "Du Pédantisme» [1580], I, 24, éd. J. Balsamo, M. Magnien et C. Magnien-Simonin, Paris, Gallimard, coll. «Bibliothèque de la Pléiade», 2007, p. 138 : Montaigne se demande si le discrédit public des professeurs est justifié ou s'il relève du stéréotype outrancier. Voir K. Kupisz, "Variations montaigniennes sur le thème du pédantisme », Revue d'Histoire littéraire de la France, 88 année, n 5, Montaigne 1588-1988 (sept.-oct. 1988), p. 944-948; T. Gontier, « Prudence et sagesse chez Montaigne ", Archives de Philosophie, 2012/1 (t.75), p. 113-130. Disponible sur <https:// www.cairn.info/revue-archives-de-philosophie-2012-1-page-113.htm>.

38. P. Larivey, Le Laquais, éd. M. Lazard et L. Zilli, Paris, Nizet, 1987, p. 34 (introduction).

39. Voir L. Sozzi, ouvr. cité, p. 382. Le personnage est la «bête noire » de Des Périers : « [...] tout un groupe de textes concerne des présomptueux et des vaniteux qui affichent des connaissances qu'ils n'ont pas (VI, XX, XXI, XXII, XXX, XL, LIX, LXVI) ou bien qui exigent un respect dont ils ne sont pas dignes (XI, XXX, XLIV, LXVIII).» (Ibid.)

40. D. Pirovano, éd. citée, IV, 4, p. 595-606.

41. Des Périers, ouvr. cité, Nouvelle XXI. Voir L. Sozzi, ouvr. cité, p. 177.

42. " [...] il captura trois cents renards, prit des torches et, tournant les bêtes queue contre queue, il plaça une torche entre les deux queues, au milieu. Il mit le feu aux torches, puis lâchant les renards dans les moissons des Philistins, il incendia aussi bien les gerbes que le blé sur pied et même les vignes et les oliviers. » (Jg 15, 4-5)

43. Pour prendre un exemple dans le théâtre de Pierre de Larivey: à une servante qui lui demande «Qui êtes-vous donc? ", le précepteur Lucian répond sans sourciller : « Philosophe, hoc est savant, docte et très éloquent. » Les docteurs florentins pourraient faire ce type de réponse. Voir Le Laquais, IV, 4, éd. citée, p. 175. La pièce a pour modèle italien Il Ragazzo de Lodovico Dolce.

44. «[...] mal habiles, peu sages et prudents, tellement qu'il semble que la science ne leur serve que de les rendre plus sots, mais encore plus arrogants, caquetteurs [...]. » (P. Charron, ouvr. cité, p. 61). Chez le continuateur de Montaigne, le tableau est complet: le pédagogue est vu comme quadruple imposteur: à la fois faux savant (doctus cum libro, "la mémoire pleine du savoir d'autrui »), faux sage («peu sages et prudents»), inutile à la société car inapte à la vie pratique (« malhabiles », « sots ») et enfin, érudit imbu de sa science.

45. Sur cette orientation « populiste» dans la tradition narrative médiévale et renaissante, voir L. Sozzi, ouvr. cité, p. 392.

46. 1 Cor 27. 
47. Même constat à propos de Des Périers chez L. Sozzi (ouvr. cité, p. 389).

48. On peut, là encore, faire un rapprochement avec la fable précédente du recueil de Straparola. De même que les docteurs de Bergame (dont on ne nous dit pas où ils ont été formés) sont vraiment savants, de même le fils du laboureur interrogé par Papiro Schizza a réellement tiré profit des années passées à Padoue : son père n'a pas jeté son argent par les fenêtres, comme tend à le faire accroire le prêtre.

49. IX, 5, p. 213. Ital. : "Non stette molto che venne una fanticella, la qual in verità era monaca, donna molto saputa e dottrinatta [...]. [...] e si misero a ragionar con esso lei. La quale poscia che ebbe parlato di molte cose, tuttavia latinamente, entrò nella teologia, e tanto catolicamente parlò, che non vi fu veruno, che non la comendasse molto. Mentre la fanticella ragionava, venne un, vestito da fornaio, tutto di carboni tinto, e intesa la disputazione che facevano con la fantesca, s'interposse e con tanta scienza e con tanta dottrina interpretò la scrittura sacra, che tutti e' dottori firentini tra sé affermavano non aver per lo adietro mai udito meglio. » (p. 611) Nous soulignons.

50. On peut faire le parallèle avec les "disputes » de palais et les joutes ludiques qui se développent en même temps que la civilisation de cour dans l'Europe du xvi siècle. À la table de François $\mathrm{I}^{\mathrm{er}}$, par exemple, les savants sont bienvenus à condition qu'ils se plient à l'élégance de la discussion polémique sans se prendre au sérieux. Voir G. Gadoffre, La Révolution culturelle dans la France des humanistes, Genève, Droz, 1997, p. 192-198.

51. «[...] la superbia, fondamento di tutti e' mali e radice d'ogni umano errore, percioché l'uomo ignorante si presume sapere quel non sa; e vuole apparere quel che non è [...]. » (« [...] la superbe, fondement de tous les maux et racine de toute erreur humaine, parce que l'homme ignorant prétend savoir ce qu'il ne sait pas, et veut apparaître tel qu'il n'est pas [...]. ») (Straparola, IX, 4, éd. D. Pirovano, p. 595).

52. Les marchands florentins ont commencé par insulter leurs homologues bergamasques en ces termes : "Vraiment, à ce que pouvons comprendre, vous êtes hommes tant lourds et grossiers que, si ce n'était le peu de trafic de marchandise que exercez, vous ne seriez bons à chose du monde; et encore que la fortune vous soit aucunement favorable en votre marchandise, si est-ce que ce n'est pour la gentillesse de votre esprit, ni par science qui soit en vous, mais plutôt par un ardent désir de gagner, et pure avarice qui vous maîtrise. Aussi êtes-vous les plus ignares de la terre. » (p. 210) Ital. : « Veramente voi Bergamaschi, per quanto noi potiamo comprendere, siete huomini tondi, e grossi, e si non fusse quella poca mercatantia, che voi fate, voi non sareste buoni di cosa alcuna per la vostra tanta grossezza. Et avenga che la fortuna vi sia favorevole nella mercatantia, non già per sottigliezza d'ingegno, ne per scienza, che voi habbiate, ma piu tosto per la ingordigia, e per l'avaritia, che dentro di voi si riserba di guadagnare, nondimeno io non conosco huomini piu grossi, ne piu ignoranti di voi.» (p. 608)

53. Catherine de Sienne ( $x v^{\mathrm{e}}$ siècle), grande figure féminine de l'ordre des dominicains, a convaincu le pape de quitter Avignon pour Rome et a marqué la pensée spirituelle et théologique avec son Dialogue ; elle fut canonisée par le pape Pie II au XV siècle (1461).

\section{RÉSUMÉS}

Dans la fable IX, 5 des Facétieuses Nuits (Le Piacevoli Notti, 1553), la référence évangélique est explicite à propos d'un bon tour joué par les Bergamasques à des Florentins érudits. Se demander dans quelle mesure la référence paulinienne alléguée s'applique vraiment à l'histoire oblige à un 
détour du côté des pédants ridicules au théâtre. Ce faisant, nous étudierons la manière dont Straparola articule dans ce conte trois motifs, dont deux ont des résonances bibliques, et comment il les renouvelle : à savoir (1) la victoire du faible sur le fort; (2) l'humiliation de l'arrogant et la supériorité paradoxale du simple sur le savant ; (3) la compétition entre cités sous la forme d'une joute oratoire.

In the 5th tale of the 9th Night from The Pleasant Nights (1553), the evangelical reference is utterly explicit, regarding a scheme used by people of Bergamo against learned Florentines. If we wonder to what extent the exhibited paulinian reference applies to the tale, a detour towards the ridiculous pedants on stage is necessary. We will also try to understand how Straparola manages to combine three components in this tale-two of which have a biblical echo-and how he renews them: namely, (1) the victory of the weak over the strong; (2) the humiliation of overconfident people and the paradoxical superiority of the common man over the learned one; (3) a dispute engaged by two rival cities.

\section{INDEX}

Mots-clés : Straparola, Les Facétieuses Nuits, Paul de Tarse, dispute (disputatio), pédantisme, "Commedia erudita », Des Périers, latin, ruse

Keywords : Straparola, The Pleasant Nights, Paul the Apostle, dispute (disputatio), pedantry, erudite comedy, Des Périers, latin, ploy

\section{AUTEUR}

\section{PATRICIA EICHEL-LOJKINE}

Le Mans Université, 3LAM 